\title{
Avanzando hacia la igualdad de oportunidades en la inclusión socio-laboral de las personas con discapacidad
}

\section{Progressing toward equal opportunity in social and occupacional inclusion of person with disabilities}

\author{
Esther MERCADO GARCÍA \\ Universidad de Castilla-La Mancha \\ esther.mercado@uclm.es \\ Eva AizPuRúa GonZÁLEZ \\ Universidad de Castilla-La Mancha \\ Eva.Aizpurua@uclm.es \\ Luis Mariano GARCíA VICENTE \\ Universidad Complutense de Madrid \\ luimgarc@trs.ucm.es
}

Recibido: 03/07/2012

Revisado: 23/10/2012

Aceptado: $17 / 12 / 2012$

Disponible on line: 16/04/2013

\begin{abstract}
Resumen
En las últimas décadas se han alcanzado notables avances en el reconocimiento de los derechos de las personas con discapacidad. Bajo esta premisa, la superación de las desigualdades persistentes en la esfera productiva se ha erigido en un objetivo preferente, dado el potencial integrador que representa el empleo. En el presente trabajo se analiza la posición que ocupa la población con discapacidad en el mercado laboral español, atendiendo a las diversas variables que configuran y moderan dicha situación. De igual modo, se observan y sintetizan los principales factores que obstruyen y limitan la inserción de las personas con discapacidad en el ámbito productivo, concretando sus fundamentos y posibles vías de superación. Finalmente, se perfilan los itinerarios de inserción laboral fundamentales, focalizando la atención en el empleo con apoyo, como metodología de intervención de eficacia contrastada, armoniosa con los principios de igualdad de oportunidades y no segregación, preeminentes en el marco normativo español. Concluye el artículo precisando las implicaciones teóricas y metodológicas que emanan del análisis efectuado, que aconsejan la articulación de actuaciones integrales que posibiliten la inclusión en las diversas esferas sociales, abogando por los postulados de transversalidad y coordinación.
\end{abstract}

Palabras clave: discapacidad, empleo con apoyo, inserción laboral, inclusión social, política social.

\begin{abstract}
Over the last few decades, significant progress has been made with regard to recognition of the rights of people with disabilities. In this context, and given the potential offered by employment in terms of integration, overcoming the inequalities which still prevail in the productive sector has become a primary aim. For that reason, this study analyses the position of disabled people in the Spanish labour market, paying attention to the diverse variables which shape and influence that position. At the same time, reference is made to the main factors which obstruct and limit the integration of the people with disabilities in the employment sector. These factors are synthesized and their causes and possible ways in which they may be overcome are established. Finally, the fundamental itineraries of integration in the world of work are outlined, focusing attention on Supported Employment as a means of intervention which has been shown to be efficient and in line with the principles of equality of opportunities and non-segregation which are preeminent in the Spanish regulatory framework. The article concludes by underlining the theoretical and methodological implications of the analysis, which stress the need for integrated action which permit inclusion in the different social spheres, and recommending the principles of mainstreaming and coordination.
\end{abstract}

Keywords: disability, supported employment, occupational integration, social inclusion, social policy.

Referencia normalizada: Mercado García, E., Aizpurúa González, E. y García Vicente, L. M. (2013): «Avanzando hacia la igualdad de oportunidades en la inclusión socio-laboral de las personas con discapacidad». Cuadernos de Trabajo Social, 26(1): 95-104.

Sumario: Introducción. 1. La situación de las personas con discapacidad en el ámbito laboral. 2. Elementos obstructores de la inserción laboral de las personas con discapacidad. 3. Fórmulas de integración laboral. El Empleo con Apoyo. 4. Conclusiones. 5. Referencias bibliográficas. 


\section{Introducción}

La discapacidad ha sido objeto de una atención creciente en el curso de las últimas décadas (De Lorenzo, 2004), que se ha materializado en una intensa actividad legislativa en los planos nacional, europeo e internacional (Campoy y Palacios, 2007). Dicho interés ha sido concomitante con la transformación desplegada en la concepción del fenómeno (Samaniego, 2006; Jiménez y Huete, 2010; Mercado y García, 2010 b), así como con el incremento cuantitativo de las situaciones de discapacidad motivado, fundamentalmente, por el progresivo envejecimiento de la población (Instituto Nacional de Estadística, 2009).

En este sentido, la noción de discapacidad y las connotaciones y significados vinculados a ella no han permanecido invariables, sino que han ido reformulándose con el trascurso del tiempo (Samaniego, 2006; American Association on Intellectual and Developmental Disabilities, 2009; Barranco, 2011), transitando desde una orientación eminentemente individualizadora - a la que subyacían conceptualizaciones animistas y clínicas - hasta el modelo bio-psico-social, imperante en la actualidad, consagrado con la aprobación de la Clasificación Internacional del Funcionamiento, de la Discapacidad y de la Salud en 2001 (Pérez, 2010; Meseguer y Montero, 2011). De este modo, se consolidó el desplazamiento de la causalidad lineal amparada previamente, a la concepción multidimensional y holística del fenómeno que se explica aludiendo a la interacción bidireccional entre la persona y el contexto social en el que participa.

Tal y como evidencian los datos procedentes de las instancias oficiales y los estudios empíricos, las personas con discapacidad constituyen un amplio y heterogéneo sector poblacional (Díaz, 2009; Mercado y García, 2010a; Jones, 2011; World Health Organization, 2011). Así, la Encuesta de Discapacidad, Autonomía Personal y Situaciones de Dependencia (en lo sucesivo EDAD) conducida en 2008, cifraba en 3.847.900 las personas con discapacidad residentes en España, suponiendo el 8,16 por ciento de la población total.

No obstante, el análisis de los resultados revela que la prevalencia de la discapacidad no es uniforme, sino que varía atendiendo a diversos factores. Observando la distribución por sexos, se constata que el 59,8 por ciento de quienes se encuentran en esta situación son mujeres, siendo las tasas de discapacidad masculina superiores hasta el tramo comprendido entre los 35 y los 44 años, en el que se invierte la tendencia. De igual modo, se advierte una correlación positiva entre la edad y la presencia de discapacidad, que alcanza su cenit en el intervalo de sujetos mayores de ochenta años.

\section{La situación de las personas con discapa- cidad en el ámbito laboral}

Dado su potencial socializador y los ingresos que de él se desprenden y que facultan el esta-

\section{$\square$ Hombres $\square$ Mujeres}

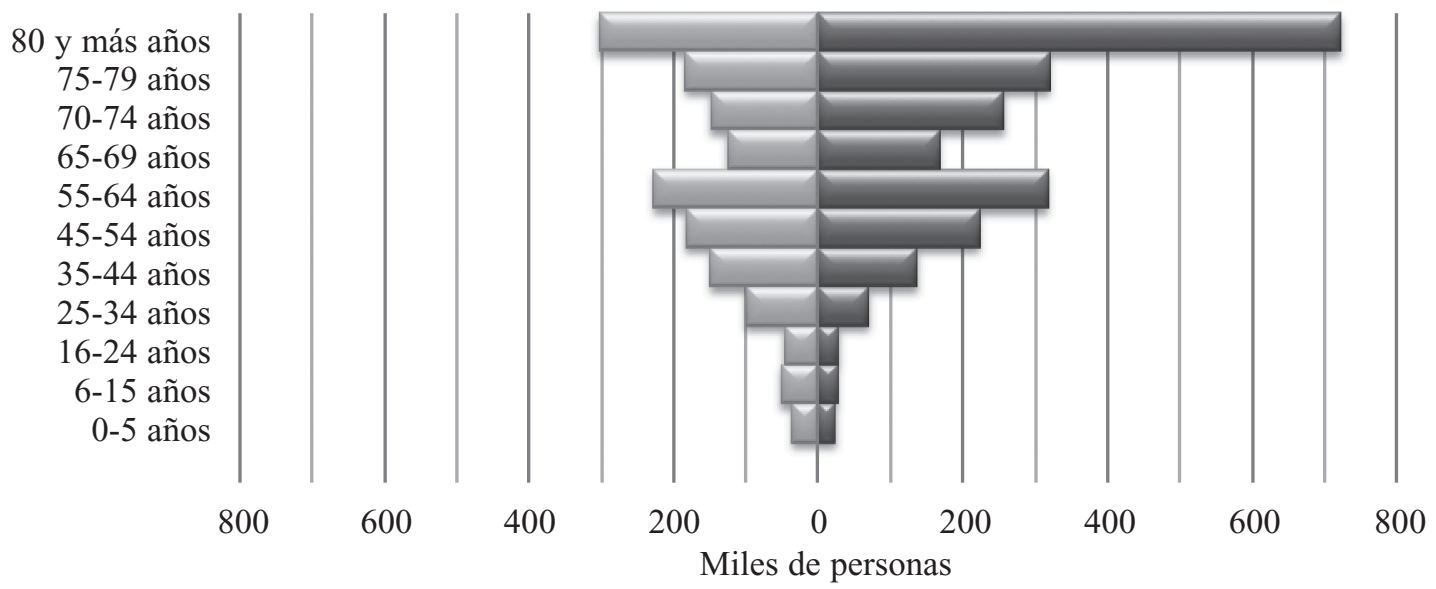

Gráfico 1. Personas con discapacidad, por sexo y grupos de edad.

Fuente: Elaboración propia, a partir de datos de la EDAD, 2008. 
blecimiento de una vida autónoma, el empleo se ha erigido, en la sociedad contemporánea, en un elemento decisivo en el proceso de integración social y participación comunitaria de la ciudadanía (Hartnett, Stuart, Thurman, Loy y Batiste, 2001; Villa y Arnau, 2003; De Lorenzo, 2004; Cazallas, 2005; Pallisera y Rius, 2007 Ali, Schur y Blank, 2011).

Desde el último tercio del pasado siglo, los organismos estatales y supranacionales han recurrido a la promulgación de disposiciones normativas orientadas a la promoción de la igualdad de oportunidades en el acceso al mercado laboral de las personas con discapacidad. Dicho desarrollo legislativo ha sido creciente y ha dejado constancia de la evolución conceptual y simbólica que ha experimentado la discapacidad (Jiménez y Huete, 2010), cuyo principal exponente ha sido la transición de medidas asistencialistas al despliegue de políticas activas de inserción laboral, así como la incorporación del principio de no discriminación como elemento transversal en el marco de las políticas públicas (Cueto, 2008; Ministerio de Trabajo e Inmigración, 2008; Ospina, 2010, World Health Organization, 2011).

Pese a la articulación de un marco jurídico garante de la igualdad formal, los hallazgos dimanantes de la literatura comparada constatan, sistemáticamente, la pervivencia de desigualdades significativas en el acceso al mercado de trabajo de este colectivo (Observatorio Ocupacional del Servicio Público de Empleo Estatal,
2008; Kaye, Jans y Jones, 2011; Pagán, 2011; World Health Organization, 2011).

Muestra de ello son los elevados índices de inactividad y desempleo que atesoran las personas con discapacidad, y que distan sustancialmente de los que posee la población carente de ella. De este modo, se advierte que en 2010, su tasa de actividad se ubicaba 39,7 puntos porcentuales por debajo de la registrada por la población general (Instituto Nacional de Estadística, 2011).

No obstante, los datos revelan que la participación en la esfera laboral se encuentra mediada por múltiples variables. Así, las desigualdades de género observadas globalmente, encuentran reflejo en este colectivo, en el que la tasa de empleo de los varones en 2010 superaba, en 8,1 puntos, la registrada por las mujeres. De igual modo, se constata que el número de personas en situación de inactividad se incrementa notablemente con la edad, comprendiendo al 71,1 por ciento de los sujetos mayores de 44 años (ídem).

Atendiendo a la tipología de discapacidad, cabe indicar que las asociadas a deficiencias auditivas y visuales acumulan las puntuaciones de actividad más elevadas $(47,5$ por ciento y 37,8 por ciento, respectivamente), en contraste con las intelectuales y aquellas vinculadas al trastorno mental, que obtienen las menores proporciones (18,2 por ciento y 16,3 por ciento, respectivamente). La intensidad de la discapacidad constituye también un elemento de es-

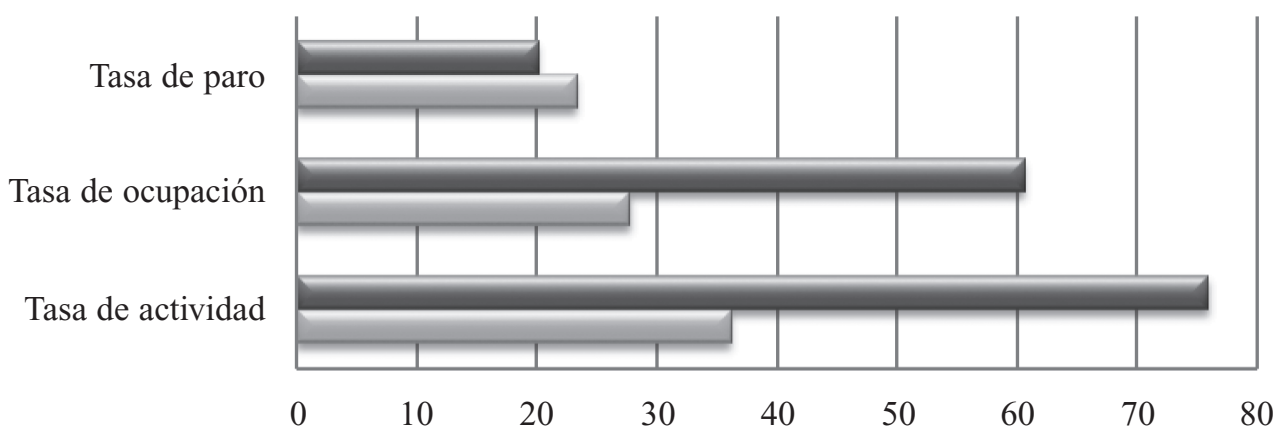

\begin{tabular}{|l|c|c|c|}
\cline { 2 - 4 } \multicolumn{1}{c|}{} & Tasa de actividad & Tasa de ocupación & Tasa de paro \\
\hline$\square$ Personas sin discapacidad & 75,9 & 60,6 & 20,1 \\
\hline$\square$ Personas con discapacidad & 36,2 & 27,7 & 23,3 \\
\hline
\end{tabular}

Gráfico 2. Tasas de actividad, empleo y paro de la población de 16 a 64 años.

Fuente: Elaboración propia, a partir de datos del Instituto Nacional de Estadística, referidos a 2010. 


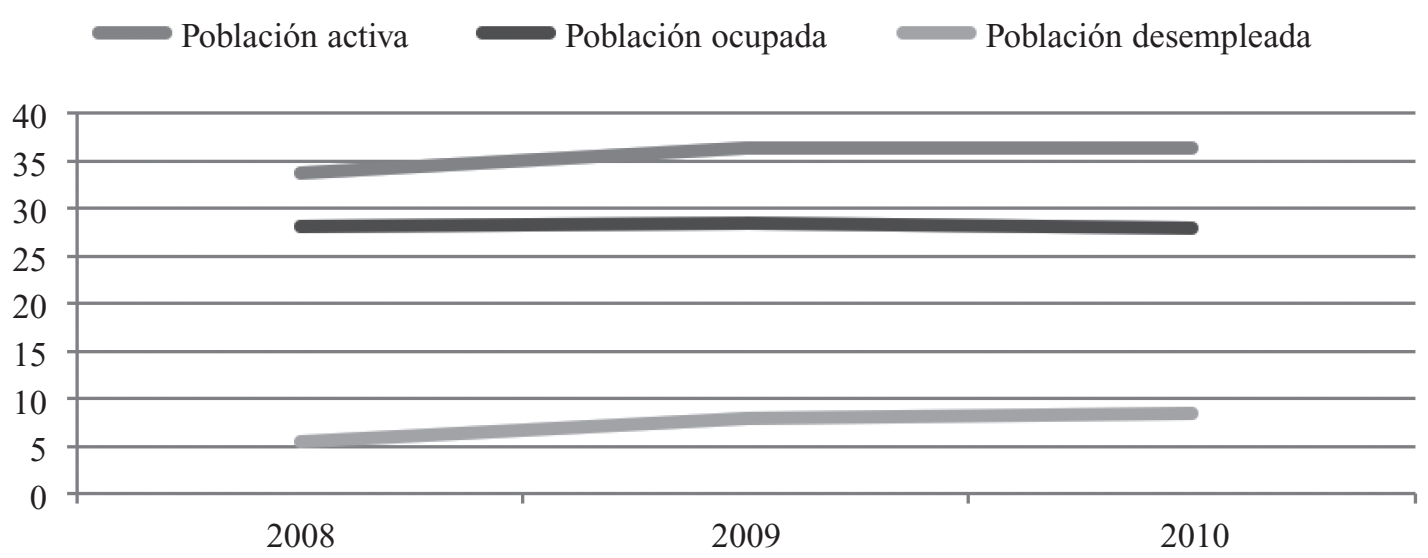

Gráfico 3. Evolución de la participación de las personas con discapacidad en el mercado laboral durante el intervalo 2008-2010.

Fuente: Elaboración propia, a partir de datos del Instituto Nacional de Estadística, referidos a 2010.

pecial trascendencia condicional de la implicación en el mercado laboral. De conformidad con los datos disponibles, a medida que aumenta el grado de discapacidad reconocida, se reducen los índices de actividad, restringiéndose significativamente en aquellas personas que poseen una graduación igual o superior al 75 por ciento (ídem).

Igualmente, se observa una correspondencia entre el nivel de instrucción y la situación de actividad, erigiéndose la formación en uno de los factores determinantes de la incorporación en el ámbito productivo. Así lo evidencia el hecho de que en 2010, quienes poseían estudios superiores alcanzaran una tasa de actividad 23,5 puntos por encima de la cifra media (ídem).

Finalmente, ha de subrayarse que la percepción de subsidios y pensiones constituye también un elemento vinculado a la situación de actividad de las personas con discapacidad. Así, se observa que el 91 por ciento de quienes recibieron alguna retribución estaban inactivas, mientras que sólo el 38,2 por ciento de quienes no tuvieron acceso a ellas se encontraba en situación de inactividad (ídem).

En lo que respecta al desempleo, es reseñable que los índices de paro correspondientes al colectivo de personas con discapacidad superaban, en 3,2 puntos porcentuales, los registrados por la población general, habiéndose incrementado 2,9 puntos desde 2008 a 2010. Y ello es así, en tanto que durante este período, la evolución de la actividad ha sido creciente, no habiéndose acompañado de un incremento equivalente de la ocupación.

La distribución de las personas desempleadas tampoco denota un patrón homogéneo, apreciándose divergencias atendiendo al sexo y la edad de referencia. Así, las mujeres exhiben, nuevamente, una mayor vulnerabilidad, sosteniendo puntuaciones más prominentes que sus homólogos varones en materia de desempleo $(24,9$ por ciento frente a 22,3 por ciento, respectivamente). La población con edades comprendidas entre los 16 y los 24 años presenta tasas de paro más elevadas que los restantes grupos de edad $(51,7$ por ciento, en contraste con 25,5 por ciento para aquellas personas ubicadas en el intervalo de 25 a 44 años, y con la cifra de 18,2 por ciento que corresponde a los sujetos mayores de 45 años).

Sin embargo, la posición de desigualdad que singulariza a las personas con discapacidad no se restringe a sus tasas de actividad, desempleo y ocupación, sino que envuelve sus condiciones contractuales, que, a su vez, supeditan la remuneración percibida, así como la tipología de jornada suscrita (Red2Red Consultores, 2009).

Tal y como atestiguan los datos, la generalidad de contratos formalizados por este sector poblacional posee una duración determinada. De este modo, se halla que el conjunto de contratos eventuales, de interinidad, por obra o servicio y temporales destinados a personas con discapacidad suponen el 76,2 por ciento del total de convenios refrendados por este co- 
lectivo durante el mes de marzo de 2012 (Observatorio Estatal de la Discapacidad, 2012).

Si bien es cierto que, en el intervalo del último año, la contratación de este sector poblacional ha decrecido globalmente un 7,77 por ciento, la disminución más prominente ha correspondido a los contratos indefinidos, cuya variación relativa de 2011 a 2012 ha sido de 24,33 puntos porcentuales (ídem).

Al igual que acontece en el plano general, las mujeres con discapacidad ostentan una mayor presencia en el empleo a tiempo parcial. Aspecto éste que reafirma su situación de mayor fragilidad estructural, en tanto que la retribución salarial y las posibilidad de promoción profesional asociadas a esta tipología de jornada son menores que las vinculadas a la contratación a tiempo completo (ídem).

Todo ello pone de manifiesto las desigualdades que experimenta la población con discapacidad en el acceso al sector productivo, que fluctúan y se agudizan atendiendo a la tipología e intensidad de la deficiencia padecida, al sexo y la edad de los sujetos y cuya permanencia obedece a un cúmulo de factores recíprocamente relacionados (Verdugo, Jenaro y Campo, 2009).

\section{Elementos obstructores de la inserción laboral de las personas con discapacidad}

En consonancia con lo apuntado, los rasgos definitorios de la posición que albergan las personas con discapacidad en la esfera laboral encuentran su origen en un conjunto de elementos, tanto micro como macro-estructurales, recíprocamente relacionados (Saunders, Leahy, McGlynn y Estrada, 2006; Ministerio de Trabajo e Inmigración, 2008; Fabian, Beveridge y Ethridge, 2009).

Entre ellos, el menor nivel formativo y la cualificación profesional fragmentaria o disonante con las demandas del sistema productivo, se erigen como elementos mantenedores de las dificultades de obtención y conservación de un empleo remunerado (Villa y Arnau, 2003; Red2Red Consultores, 2009; Khudorenko, 2011). En 2008, las personas con discapacidad desprovistas de estudios primarios superaban, ampliamente, a la población general. A excepción de las enseñanzas de grado medio, en las que su presencia prevalecía, en los restantes niveles de educación superior su posición era minoritaria (Instituto Nacional de Estadística, 2009).
Por ello, la promoción de su plena inclusión en los diversos niveles que integran el sistema educativo se ha instituido en objetivo preeminente de las políticas públicas contemporáneas (Ministerio de Sanidad y Política Social, 2009; Webb, Webb y Fults-McMurtery, 2011).

A las carencias formativas se agrega la pervivencia de estereotipos y prejuicios sociales vinculados al potencial laboral de la población con discapacidad que restringen sus posibilidades de acceso al mercado de trabajo (Ministerio de Trabajo e Inmigración, 2008; Kaye, Jans y Jones, 2011; Khudorenko, 2011).

Igualmente, los déficits de accesibilidad de los espacios físicos constituyen un hándicap medular, inhibidor de la incorporación de las personas con discapacidades de carácter físico y sensorial al sector productivo (Villa y Arnau, 2003; De Lorenzo, 2004; Red2Red Consultores, 2009). Junto a ello, cabe recalcar los perjuicios derivados del incumplimiento de las medidas de acción positiva amparadas normativamente, cuya inobservancia va en detrimento de la equiparación de oportunidades de este colectivo (Ministerio de Trabajo e Inmigración, 2008; Mercado y García, 2010a), así como la subsistencia de políticas pasivas, cuya aplicación actúa como elemento disuasivo en la búsqueda y el mantenimiento de un empleo remunerado (Red2Red Consultores, 2009 Ali, Schur y Blank, 2011).

A los factores anteriormente expuestos se vinculan dos aspectos de especial trascendencia que recrudecen, aún más, la situación de precariedad de las personas con discapacidad así como sus dificultades de integración en el mercado laboral. El primero de ellos alude a la dispersión y disgregación de los recursos de información dirigidos a este sector poblacional (Pallisera, 2011), que si bien responde a los requerimientos de especialización, obstaculiza la adquisición de una panorámica integral de los itinerarios y vías de incorporación a la esfera productiva. El segundo hace referencia a las expectativas personales y familiares con respecto a su participación activa en el mercado de trabajo (Pallisera y Rius, 2007; González, 2009; Red2Red Consultores, 2009). En consonancia con los hallazgos dimanantes de la literatura acumulada, los convencionalismos preponderantes pueden actuar como catalizadores de conductas de sobreprotección familiar y actitu- 
des de desmotivación individual, obstructoras del acceso de este sector al ámbito laboral.

Todo ello constata el carácter polifacético y multidimensional del fenómeno analizado, determinado por una pluralidad de variables recíprocamente interconectadas (Ministerio de Trabajo e Inmigración, 2008).

\section{Fórmulas de integración laboral. El em- pleo con apoyo}

En la actualidad coexisten diversas vías de integración en el mercado productivo. Así, distinguimos entre el empleo protegido, consistente en el establecimiento de una oferta laboral específica, canalizada a través de los Centros Especiales de Empleo, como paso preliminar y enlace con el empleo normalizado; y el empleo ordinario, regido por los principios de igualdad y no segregación, tendente a la inclusión directa de las personas con discapacidad en el ámbito productivo estandarizado (De Lorenzo, 2004; Observatorio Ocupacional del Servicio Público de Empleo Estatal, 2008). Si bien constituyen fórmulas divergentes, existe unanimidad en cuanto a su consideración como estrategias complementarias y no excluyentes de superación de las desigualdades imperantes (Villa, 2003; De Lorenzo, 2004).

Bajo el paradigma del trabajo ordinario se enmarca la metodología del «empleo con apoyo» (Supported Employment), que instituye un modelo de integración laboral emergido en Estados Unidos durante la década de 1980 (Verdugo, Jordán y Bellver, 1998; Arenas y González, 2010).

La traslación y aplicación de este modelo en España se ha sucedido con posterioridad, adquiriendo significación a partir de 1990 . Pese a que la proliferación de programas especializados se ha extendido por los distintos territorios del Estado, ésta no ha sido uniforme, existiendo notables diferencias en su desarrollo y consolidación territorial (Verdugo, Jordán y Bellver, 1998). No obstante, su implantación ha sido gradual, hallando reconocimiento jurídico con la promulgación del Real Decreto $870 / 2007$, de 2 de julio, por el que se regula el programa de Empleo con apoyo como medida de fomento del empleo de las personas con discapacidad en el mercado ordinario ${ }^{1}$.
En consonancia con Arenas y González (2010), este modelo metodológico tiene como objeto facilitar la incorporación laboral de quienes experimentan notables limitaciones de carácter físico, psíquico o sensorial, articulando un conjunto de procedimientos técnicos de apoyo individualizado. De este modo, el empleo con apoyo configura un sistema secuencial integrado por sucesivas fases - que comprenden la búsqueda de empleo, el estudio y valoración particularizados, el análisis del puesto de trabajo, el proceso de selección y colocación, el seguimiento y la valoración global- (Jordán, 2011), cuyos principales ejes vertebradores son la formación, la prospección laboral, el seguimiento y el afianzamiento de redes de apoyo, favorecedoras del establecimiento de una vida autónoma.

Esta metodología, fundamentada en el refuerzo de la población con dificultades de inserción en la esfera productiva, comprende actuaciones sincrónicas, caracterizadas por el tránsito de un apoyo intensivo a otro natural, en el que el rol del profesional deviene, gradualmente, menos activo y directivo, al tiempo que se potencia la autodeterminación del usuario (Arenas y González, 2010).

En armonía con las transformaciones legislativas practicadas en los últimos años, conducidas por los principios de transversalidad, normalización, empoderamiento e igualdad de oportunidades, las políticas activas en materia de empleo han adquirido una atención creciente, amplificando su alcance y ámbito de aplicación (Jordán, Verdugo y Sainz, 2012). Como parte de ellas, el «empleo con apoyo» ha experimentado un progresivo incremento que se ha materializado en la proliferación de iniciativas públicas y privadas, así como en el crecimiento sostenido de los receptores de estos programas (Becerra, Montanero y Lucero, 2011; Jordán y Verdugo, 2011).

La comunidad científica internacional ha evidenciado, con solidez, la eficacia que ofrece esta metodología como resorte para la inclusión laboral de las personas con discapacidad, tanto en términos de consecución de un puesto de trabajo, como en lo referido a la conservación del mismo (Jordán y Verdugo, 2010; Campbell, Bond y Drake, 2011).

1 A este respecto cabe indicar que en el momento de elaboración del artículo se encuentra en proceso de estudio la aprobación de un nuevo Reglamento. 
De conformidad con lo enunciado previamente, el empleo remunerado instituye un medio decisivo de integración social (Villa, 2003; De Lorenzo, 2004; Cazallas, 2005; Mercado y García, 2010a), propiciando la autonomía personal y económica (Cueto, 2008), así como erigiéndose en un espacio privilegiado de interacción social y participación comunitaria (Ministerio de Sanidad y Política Social, 2009). No obstante, diversos autores han recalcado que, pese a la significación que adquiere el trabajo en la sociedad contemporánea, la tenencia de un empleo remunerado es condición necesaria, aunque no suficiente, para alcanzar la inclusión de la persona en las diversas esferas sociales (Pallisera y Rius, 2007; Verdugo et al., 2010). Por ello, en tanto que la normativa estatal, comunitaria e internacional abogan por la plena inclusión de las personas con discapacidad, y en la medida en que su posición material y simbólica se encuentra mediada por una multiplicidad de factores interdependientes, el ámbito laboral no puede concebirse como recinto aislado e inconexo, sino que las políticas públicas y actuaciones desplegadas han der responder a una representación global y multidimensional del fenómeno.

Bajo esta premisa, el «empleo con apoyo» emerge como metodología privilegiada, cuyos resultados constatan su suficiencia como herramienta eficaz de inserción laboral, de acuerdo a los principios rectores de normalización e igualdad de oportunidades, contribuyendo a la superación de las desigualdades que tolera este amplio y heterogéneo colectivo.

\section{Conclusiones}

La revisión efectuada faculta el establecimiento de tres conclusiones fundamentales, emanadas del análisis del estado actual de la cuestión.

La primera de ellas, constata la posición de desigualdad que experimentan las personas con discapacidad en el ámbito laboral y cuya existencia obedece a un conjunto de factores personales, familiares y estructurales recíprocamente relacionados (Saunders, Leahy, McGlynn y Estrada; Ministerio de Trabajo e Inmigración, 2008; Fabian, Beveridge y Ethridge, 2009). Dado el carácter heterogéneo de este sector poblacional, dicha circunstancia se recrudece atendiendo a determinadas variables, tales como el sexo, la edad, la tipología de deficiencia padecida, la intensidad de la discapacidad y el nivel de instrucción. De este modo, las dilatadas tasas de inactividad y desempleo que singularizan a este colectivo, afectan, con mayor severidad, a las mujeres, los sujetos más jóvenes, a quienes poseen deficiencias intelectuales y albergan menor formación, así como a aquellas personas con un grado de discapacidad superior al 75 por ciento (Encuesta de Discapacidad, Autonomía Personal y Situaciones de Dependencia, 2008; Instituto Nacional de Estadística, 2011; Observatorio Estatal de la discapacidad, 2012). La resonancia e implicación práctica de dicha coyuntura alude a la conveniencia de articular medidas concretas de acción positiva dirigidas a subsanar la situación de mayor vulnerabilidad experimentada por tales grupos, armonizándolas con las actuaciones globales tendentes a la protección y el patrocinio de la igualdad de oportunidades.

En segundo término, cabe recalcar la evolución experimentada por el concepto de discapacidad y los atributos a él vinculados, cuyo reflejo fundamental ha sido el tránsito de políticas pasivas al establecimiento de fórmulas activas de inserción laboral (Cueto, 2008; Pérez, 2010; Jiménez y Huete, 2010; Barranco, 2011; World Health Organization, 2011; Meseguer y Montero, 2011). Bajo este modelo, la metodología del «empleo con apoyo» ha adquirido, en el curso de las últimas décadas, un notable crecimiento, tanto en la esfera nacional como en el marco internacional (Jordán y Verdugo, 2010; Becerra, Montanero y Lucero, 2011; Jordán, Verdugo y Sainz, 2012). Su correspondencia con los principios de normalización y no segregación, amparados por la legislación contemporánea, así como su eficacia en la promoción del acceso y mantenimiento de un empleo remunerado, han erigido esta metodología en herramienta contrastada de actuación.

Finalmente, pese a la relevancia del trabajo como procedimiento propulsor de la integración social de las personas, diversas investigaciones han evidenciado que la ocupación no es garante, en sí misma, de la inclusión social, precisándose de la articulación simultánea de medidas orientadas al afianzamiento de dicho proceso (Pallisera y Rius, 2007; Verdugo et al., 2010), que den cumplimiento a los requerimientos de actuación integral y coordinada, motivados por el carácter multifactorial y com- 
plejo del fenómeno analizado. En este sentido, las actuaciones individuales y familiares consagradas a la promoción de la inserción educativa y laboral, y, por ende, a la autonomía personal y económica de las personas con discapacidad, habrán de armonizarse con políticas sociales tendentes a la superación de los prejuicios y estereotipos imperantes, así como con el despliegue de instrumentos de supervisión que avalen el cumplimiento de las disposiciones normativas. Garantizando, así, la intervención sobre los múltiples condicionantes que determinan la posición de las personas con discapacidad en la realidad social.

\section{Referencias bibliográficas}

Ali, M., Schur, L. y Blank, P. (2011). What types of Jobs do people with disabilities want? Journal of Occupational Rehabilitation, 21 (2), 199-210.

American Association on Intellectual and Develpmental Disabilities. (2009). Intellectual Disability. Definition, Classification, and System of Supports. Washington: AAIDD.

Arenas, M.L. y González, R. (2010). El preparador laboral en el Empleo con Apoyo. Educación y Futuro, 23, 43-50.

Barranco, M.C. (coord.) (2011). Situaciones de dependencia, discapacidad y derechos. Madrid: Dykinson.

Becerra, M.T., Montanero, M. y Lucero, M. (2011). Empleo con apoyo. Apoyo laboral a trabajadores con discapacidad intelectual en tareas que requieren autorregulación. Campo Abierto, 30 (2), 43-63.

Campbell, K., Bond, G.R. y Drake, R.E. (2011). Who benefits from supported employment: a meta-analitic study. Schizophrenia Bulletin, 37 (2), 370-380.

Campoy, I. y Palacios, A. (2007). Igualdad, no discriminación y discapacidad. Una visión integradora de las realidades española y argentina. Madrid: Dykinson.

Cazallas, C. (2005). La desigualdad en el mercado de trabajo de personas con discapacidad: Un enfoque teórico. Revista Universitaria de Ciencias del Trabajo, 6, 147- 164. Disponible en: http://www.ruct.uva.es/pdf/Revista por ciento206/6106.pdf

Cueto, M.B. (2008). Trayectorias laborales de las personas con discapacidad y Centros Especiales de Empleo: Análisis empírico con la MCVL. Madrid: Secretaría de Estado de Seguridad Social.

De Lorenzo, R. (2004). El futuro de las discapacidades en el mundo: El empleo como factor determinante para la inclusión. Revista del Ministerio de Trabajo y Asuntos Sociales, 50, 73-89. Disponible en: http://www.empleo.gob.es/es/publica/pub_electronicas/destacadas/revista/ numeros/50/Est04.pdf

Díaz, A. (2009). La inserción laboral de las personas con discapacidad en la provincia de A Coruña desde una perspectiva de género. Santiago de Compostela: Universidad de Santiago de Compostela.

Real Decreto 870/2007, de 2 de julio, por el que se regula el programa de empleo con apoyo como medida de fomento del empleo de personas con discapacidad en el mercado ordinario. Boletín Oficial del Estado, de 14 de julio de 2007, 168, 30618 - 30622.

Fabian, E.S., Beveridge, S. y Ethridge, G. (2009). Differences in perceptions of career barriers and supports for people with disabilities by demographic, background and case status factors. The Journal of Rehabilitation, 75 (1), 41-49.

González, C.G. (2009). Visión global de la cultura con respecto al trabajo en personas con discapacidad y adultos mayores. Revista de Psicología de la Universidad Autónoma del Estado de México, 2, 52- 9.

Hartnett, H.P., Stuart, H., Thurman, H., Loy, B. y Batiste, L.C. (2011). Employers' perception of the benefits of workplace accomodations: Reasons to hire, retain and promote people with disabilities. Journal of Vocational Rehabilitation, 34 (1), 17-23. DOI: 10.3233/JVR - 2010-0530.

Instituto Nacional de Estadística. (2009). Panorámica de la discapacidad en España. Encuesta de Discapacidad, Autonomía Personal y Situaciones de Dependencia. 2008. Boletín Informativo del Instituto Nacional de Estadística. Disponible en: http://www.ine.es/revistas/cifraine/1009.pdf 
Instituto Nacional de Estadística. (2011). El empleo de las personas con discapacidad. Explotación de la Encuesta de Población Activa y de la Base Estatal de Personas con Discapacidad. Madrid: Instituto Nacional de Estadística.

Jiménez, A. y Huete, A. (2010). Políticas públicas sobre discapacidad en España. Hacia una perspectiva basada en los derechos. Política y Sociedad, 1 (47), 137-152. Disponible en: http://www.um.es/discatif/documentos/PyS/9_Jimenez_Huete.pdf

Jones, M.K. (2011). Disability, employment and earnings: an examination of heterogeinity. Applied Economics, 43 (8), 1001-1017.

Jordán, F.B. (2011). Acercamiento al Empleo con Apoyo y breve análisis de España e Iberoamérica. Psicología, Conocimiento y Sociedad, 1 (4), 21-40.

Jordán, F.B. y Verdugo, M.A. (2010). Documento Blanco del INICO. El empleo integrado como meta irrenunciable. Boletín de Noticias del Instituto Universitario de Integración en la Comunidad, 13 (37), 1- 12.

Jordán, F.B. y Verdugo, M.A. (2011). Evolución del empleo con apoyo en España 1995 a 2011 y ajuste a los estándares de calidad de la EUSE. Salamanca: INICO.

Jordán, F.B., Verdugo, M.A. y Sainz, F. (2012). Active inclusion of Young people with disabilities or health problems: Spain. Dublín: European Foundation for the Improvement of Living and Working Conditions.

Kaye, H.S., Jans, L.H. y Jones, E.C. (2011). Why don't employers hire and retain workers with disabilities? Journal of Occupational Rehabilitation, 21, 526-536.

Khudorenko, E.A. (2011). Problems of the education and inclusion of people with disabilities. Russian Education and Society, 12 (53), 82- 91.

Martín M. B. (2010). Análisis y Retos del Sistema de Autonomía y Atención a la Dependencia en Andalucía en el marco de la gobernanza multinivel. Cuadernos de Trabajo Social, 23, 163-187.

Mercado, E. y García, L.M. (2010a). La inserción laboral de las personas con discapacidad: Una salida profesional para trabajadores sociales. Portularia, 1(10), 51-60. Disponible en: http://rabida.uhu.es/dspace/bitstream/handle/10272/4284/b15919031.pdf?sequence=2

Mercado, E. y García, L.M. (2010b). Necesidades sociales de las personas con discapacidad en edad escolar y sus familias. Cuadernos de Trabajo Social, 23, 9-24. Disponible en: http://www.ucm.es/BUCM/revistas/trs/02140314/articulos/CUTS1010110009A.PDF

Meseguer, L. y Montero, J.M. (2011). Discapacidad en femenino: Situación en Castilla-La Mancha. En S. Sierra y J.C. Ortiz (coords.), El derecho y la economía de las mujeres y la igualdad de género. Valladolid: Lex Nova.

Ministerio de Sanidad y Política Social. (2009). III Plan de Acción para las Personas con Discapacidad. 2009-2012. Madrid: Ministerio de Sanidad y Política Social. Disponible en: http://sid.usal.es/idocs/F8/FDO21595/III_pacd.pdf

Ministerio de Trabajo e Inmigración. (2008). Estrategia global de acción para el empleo de las personas con discapacidad. 2008-2012. Madrid: Ministerio de Trabajo e Inmigración.

Observatorio Estatal de la Discapacidad. (2012). Información estadistica sobre contratación de personas con discapacidad. Situación a 1 de abril de 2012. Madrid: Observatorio Estatal de la Discapacidad. Disponible en: http://www.observatoriodeladiscapacidad.es/sites/default/files/ Contratacion_Marzo_2012.pdf

Observatorio Ocupacional del Servicio Público de Empleo Estatal. (2008). Informe del mercado de trabajo de las personas con discapacidad. Madrid: Ministerio de Trabajo y Asuntos Sociales; INEM.

Ospina, M.A. (2010). Discapacidad y sociedad democrática. Revista Derecho del Estado, 24, 143-164.Disponible en: http://portal.uexternado.edu.co/irj/go/km/docs/documents/UExternado/pdf/5_revistaDerechoDelEstado/numero por ciento2024/MarioOspina.pdf

Pagán, R. (2011). Self-employment and job satisfaction: evidence for older people with disabilities in Europe. European Journal of Ageing, 8, 177- 187. DOI: 10.1007/s10433-011-0194-4

Pallisera, M. (2011). La inclusión laboral y social de las personas con discapacidad intelectual. El papel de la escuela. Revista Interuniversitaria de Formación del Profesorado, 5 (1), 185-200. 
Pallisera, M. y Rius, M. (2007). ¿Y después del trabajo, qué? Más allá de la integración laboral de las personas con discapacidad. Revista de Educación, 342, 329-348.

Pérez, L.C. (2010). Discapacidad, derecho y políticas de inclusión. Madrid: CINCA.

Red2Red Consultores. (2009). El empleo de las personas con discapacidad ante el nuevo paradigma de la flexiguridad. Madrid: Fundación ONCE; Fondo Social Europeo.

Samaniego, P. (2006). Aproximación a la realidad de las personas con discapacidad en Latinoamérica. Madrid: Comité Español de Representantes de Personas con discapacidad.

Saunders, J.L., Leahy, M.J., McGlynn, C. y Estrada, N. (2006). Predictors of Employment Outcomes for Persons with Disabilities: An Integrative Review of Potential Evidenced-based Factors. Journal of Applied Rehabilitation Counseling, 37 (2), 3-20.

Verdugo, M.A., Jenaro, M. C. y Campo, M. (2009). Report on the employment of disabled people in European countries. Spain. Leeds: Academic Network of European Disability Experts.

Verdugo, M.A., Jordán, F.B. y Bellver, F. (1998). Situación actual del Empleo con Apoyo en España. Siglo Cero, 175 (29), 23-31.

Verdugo, M.A., Jordán, F.B., Santamaría, M., Orgaz, B., Ruíz, N., Martín, R. y Benito, M.C. (2010). La mejora de la calidad de vida mediante el empleo con apoyo. La experiencia del programa empleo con apoyo Caja Madrid. Educación y Futuro, 23, 13-30.

Villa, N. y Arnau, M.S. (2003). Situación laboral de las personas con discapacidad en España. Revista Complutense de Educación, 2 (14), 393-424. Disponible en: http://revistas.ucm.es/ index.php/RCED/article/view/RCED0303220393A

Webb, D., Webb, T.T. y Fults-McMurtery, R. (2011). Physical Educators and School Counselors Collaborating to Foster Successful Inclusion of Students with Disabilities. Physical Educator, 68 (3), 124-129.

World Health Organization. (2011). World Report on Disability. Malta: World Health Organization. Disponible en: http://whqlibdoc.who.int/publications/2011/9789240685215_eng.pdf 